\title{
Inverted Capacitive Deionization with Highly Enhanced Stability Performance Utilizing Ionic Liquid-functionalized Carbon Electrodes
}

Xiaoping Che a , Shiyong Wang a, Changping Li ${ }^{b}$, Gang Wang ${ }^{\text {**, }}$ Chengxu Li a, Shuaifeng Wang ${ }^{\text {a, }}$ Duanzheng Li ${ }^{\text {a }}$, Jieshan Qiu ${ }^{\text {a* }}$

a State Key Lab of Fine Chemicals, School of Chemical Engineering, Liaoning Key Lab for Energy Materials and Chemical Engineering, Dalian University of Technology, High Technology Zone, No.2 Linggong RD, Dalian 116024, Liaoning, China.

b Research Center for Eco-Environmental Engineering, School of Environment and Civil Engineering, Dongguan University of Technology, No.1 Daxue RD, Dongguan 523106, Guangdong, China.

*Corresponding author: A/Prof. Gang Wang, Prof. Jieshan Qiu

E-mail: wghy1979@163.com (G Wang); jqiu@dlut.edu.cn (J.S. Qiu)

(There are 29 pages, 11 figures and no table in the manuscript. There are 5 pages, 4 figures and no table in the supporting information.) 


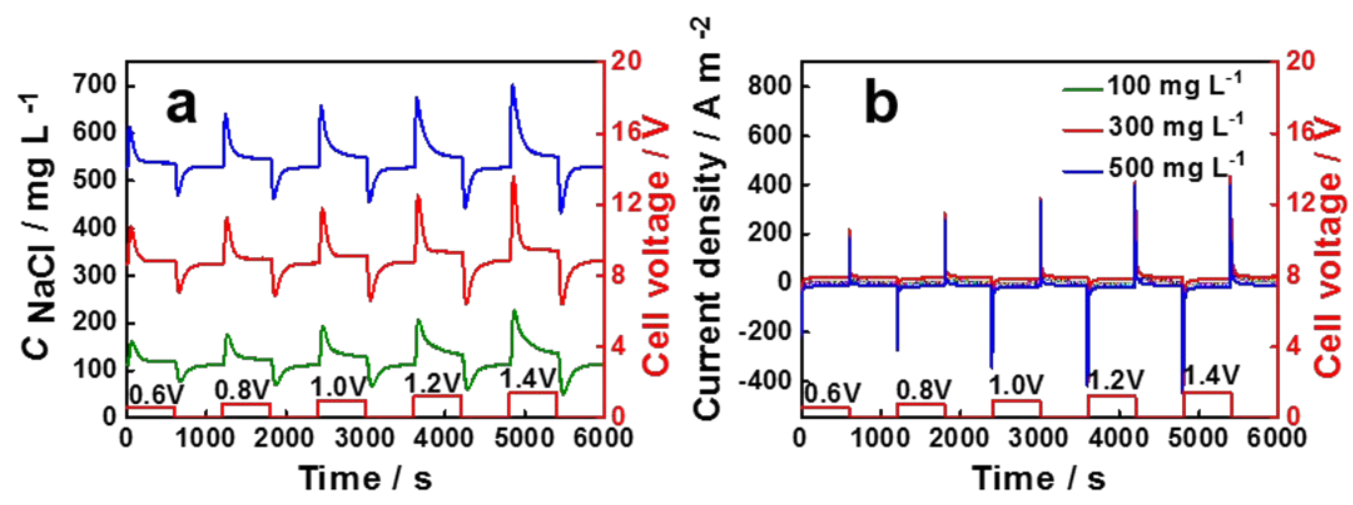

Fig. S1. (a) $\mathrm{NaCl}$ concentration changes and (b) the current of i-CDI cell with different initial $\mathrm{NaCl}$ concentrations. 



Fig. S2. Plots of conductivity and current vs. time measured during cycling of i-CDI system at the cell voltage of $0.8 / 0 \mathrm{~V}$. 

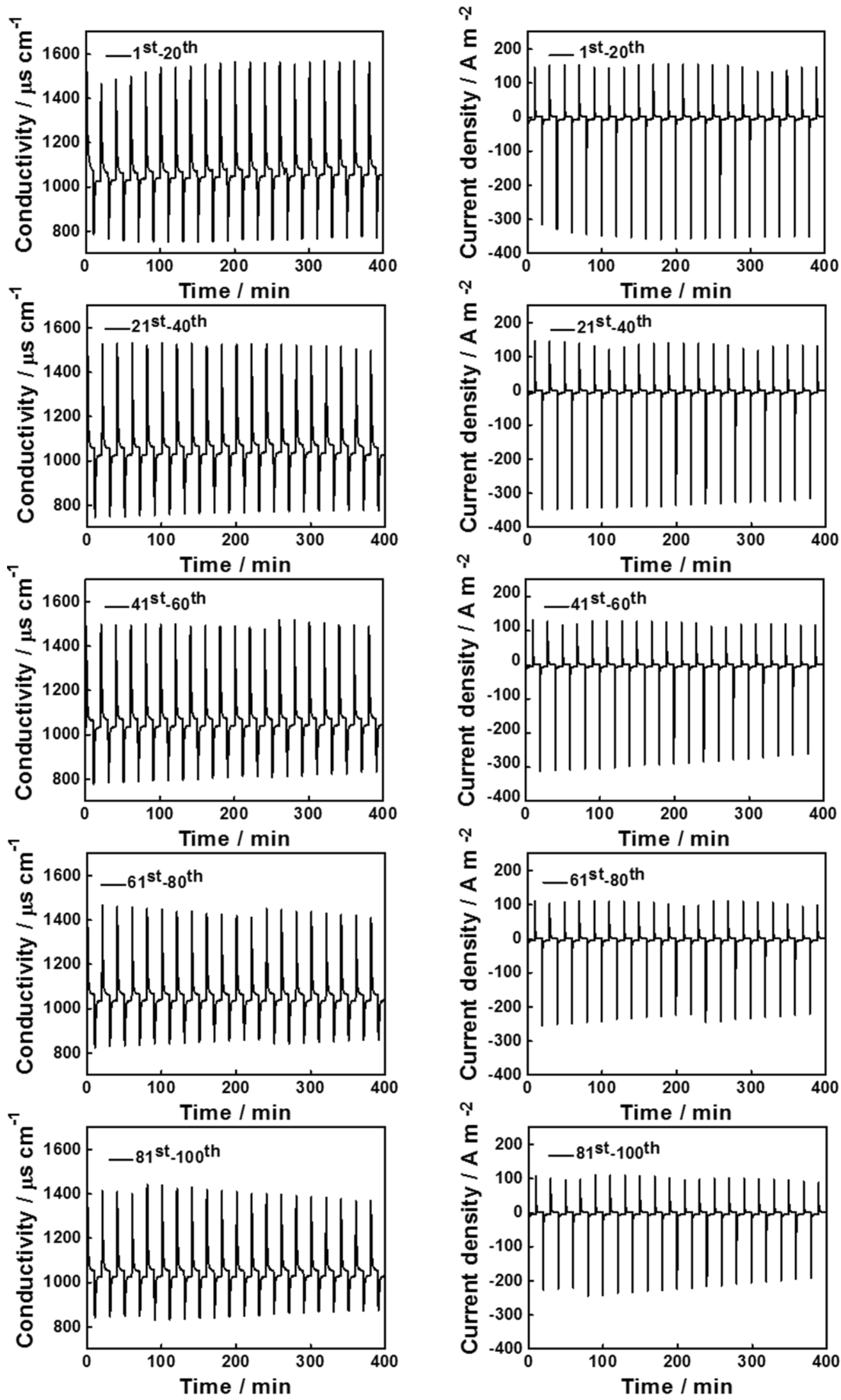

Fig. S3. Plots of conductivity and current vs. time measured during cycling of i-CDI system at the cell voltage of $1.2 / 0 \mathrm{~V}$. 

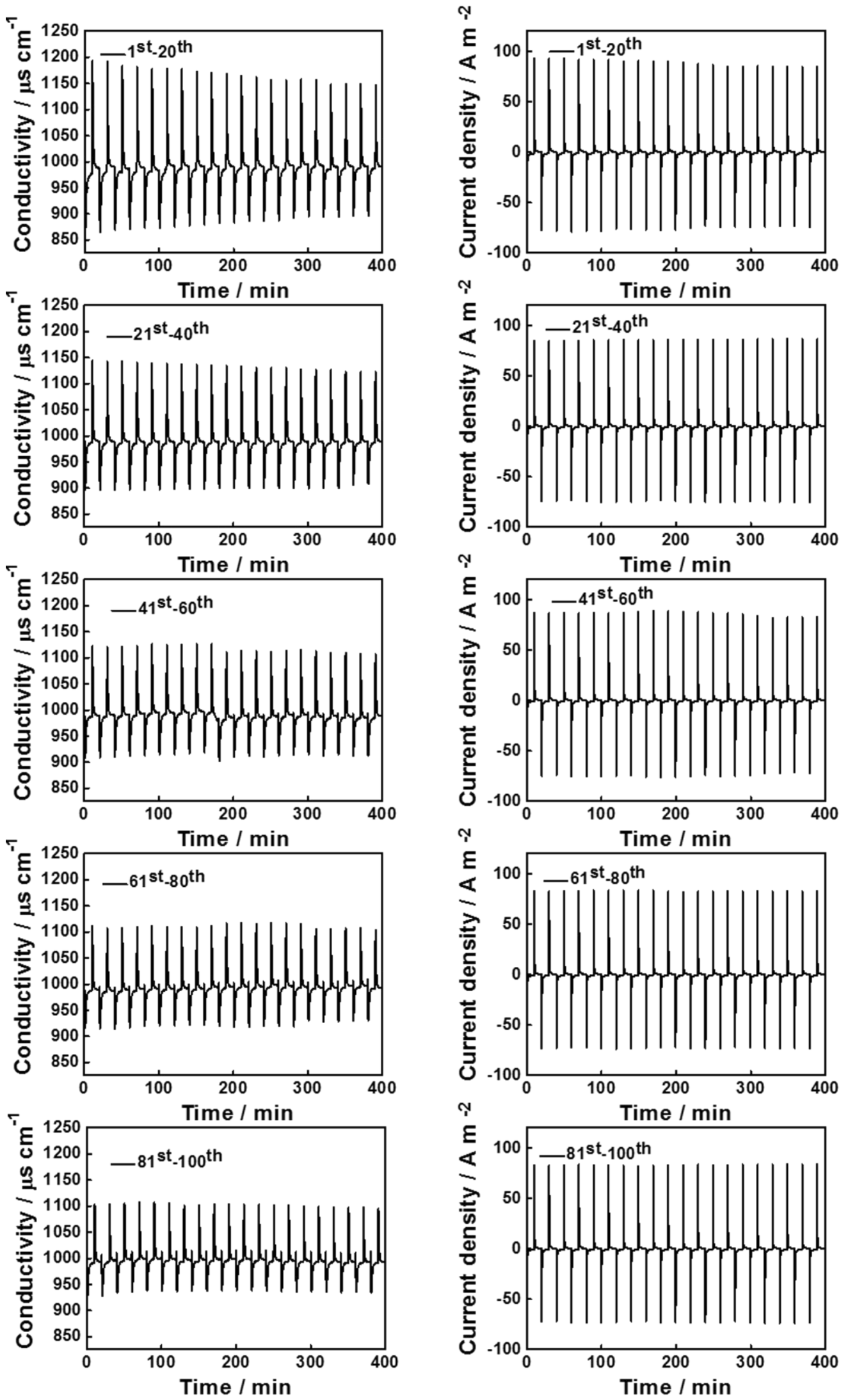

Fig. S4. Plots of conductivity and current vs. time measured during cycling of CDI system at the cell voltage of $0.8 / 0 \mathrm{~V}$ 